\title{
Study on upper limb rehabilitation system based on surface EMG
}

\author{
Lan Wang, Hailong $\mathrm{Li}^{*}$, Zhengyu Wang and Fandong Meng \\ College of Mechanical and Electrical Engineering, Harbin Engineering University, No. 145 Nantong \\ Street, Nangang District, Harbin, 150001, China
}

\begin{abstract}
During the rehabilitation process, it is essential to accurately judge a patient's recovery in a timely manner. A reasonable and matched training program is significant in the development of rehabilitation system. This paper presents a new upper limb rehabilitation training system, which consists of an upper limb rehabilitation training device, a current detection circuit, a motor speed test circuit, a surface EMG (sEMG) sensor, and a dSPACE HIL simulation platform. The real-time output torque of the servo motor is calculated by using the motor's real-time current and speed, in order to monitor the patient's training situation. The signal of sEMG is collected in real time and is processed with root mean square (RMS) to characterize the degree of muscle activation. Based on this rehabilitation system, maximum voluntary contraction (MVC) experiments, passive training experiments under different speeds, and active training experiments under different damping are studied. The results show that this new system performs real-time and accurate monitoring of a patient's training situation. It can also assess a patient's recovery through muscle activation. To a certain extent, this system provides a platform for research and development of rehabilitation medical engineering.
\end{abstract}

Keywords: Surface EMG, upper limb rehabilitation system, rehabilitation assessment

\section{Introduction}

In recent years, the number of patients with movement disorders caused by strokes has increased [1]. Rehabilitation training is a significant medical procedure that goes beyond surgery and other medical treatments to assist in patient recovery [2]. However, traditional rehabilitation training is mainly done by medical staff, thus the training will be affected by the medical staff's business level [3]. It is essential to judge a patient's recovery accurately, and to develop a reasonable and matched training program [4]. Presently, rehabilitation training can be done by using a robot instead of medical staff.

Hogan, et al. [5, 6] began the design and research of upper limb rehabilitation robots in the 1990s. An upper limb rehabilitation robot named after MIT-Manus was developed. It performed twodimensional auxiliary rehabilitation training of the shoulder and elbow in the plane. In addition, it measured and recorded the movement process with precision, and provided visual feedback through the computer. After training, it evaluated the effect of the training, combining the collected motion information with clinical evaluation methods. Reinkensmeyer, et al. [7-9] developed a rehabilitation

\footnotetext{
* Address for correspondence: Hailong Li, College of Mechanical and Electrical Engineering, Harbin Engineering University, No. 145 Nantong Street, Nangang District, Harbin, 150001, China. Tel.: +8615124571358; Fax: 054182519710; E-mail: lhl2hww@163.com.
}

0959-2989/15/\$35.00 @ 2015 - IOS Press and the authors. 
training device that could control three degrees of freedom by using a magnetic powder brake and a motor. This device can examine hand movements at different locations, and the rehabilitation effect was analyzed by comparing the actual location and the target location of patient hand movements. The training results showed that it greatly assisted with the treatment. The research team led by Loureiro, et al. $[10,11]$ developed an upper limb rehabilitation training robot that it could provide passive training, active training and impedance training. Clinical trials showed that it strongly influenced the rehabilitation of the upper limb. The rehabilitation training robot developed by Zhang, et al. $[12,13]$ executed two-dimensional rehabilitation training using two connecting rod mechanisms. The upper limb rehabilitation robot with five degrees of freedom developed by Li, et al. [14] used an exo-skeletal design and worked on the shoulder, elbow and wrist, helping the user to complete everyday tasks.

As mentioned above, there is evidence that these robots assist in patient recovery. However, they are rarely able to evaluate the effects of training effect from a patient's physical information such as the degree of muscle activation. In addition, the real-time monitoring of the patient's recovery is insufficient.

To solve the above problems, this paper proposes an upper limb rehabilitation training system based on surface EMG. The real-time output torque of the servo motor can be calculated using the motor's real-time current flow and speed. This allows the patient's training to be monitored in real time. In the time domain, the signal of sEMG is collected in real time, and it is processed with root mean square (RMS), characterizing the degree of muscle activation. Then these data are provided to medical staff or researchers as a feedback, allowing them to make an accurate judgment on the patient's actual situation. The patient is then able to improve movement function in a safe and scientific way.

\section{Upper limb rehabilitation evaluation system}

In this upper limb rehabilitation evaluation system, the real-time output torque of the servo motor is calculated with the motor's real-time current flow and the speed of the machine's end. It reflects the way in which the machine interacts with the patient. In addition, the system can also record the patient's skin surface EMG during training in real time. Rehabilitation physician and researchers have analyzed the motion information of the human upper limb and the activation degree of the upper limb muscles by monitoring real-time changes in the above data. Thus, the matched evaluations of patients' rehabilitation training under different conditions can help patients perform upper limb rehabilitation training in a more scientific way.

\subsection{Description of upper limb rehabilitation evaluation system}

The rehabilitation evaluation system consists of an upper limb rehabilitation training device, a current detection circuit, a motor speed test circuit, a sEMG sensor, and a dSPACE HIL simulation platform.

(1) Upper limb rehabilitation training device

The upper limb rehabilitation training device does both passive and active training. It can be set to different speeds in the passive exercise process, allowing patients to train at different speeds in order to exercise joint activity. In the active training process, the device can be set to different damping modes to help patients to accomplish different strength training.

(2) Current and speed test circuit 
In the current detection circuit, the output voltage of the current detection chip has a certain relationship with the current: $\mathrm{U}=2.5 \mathrm{~V}+0.185 \mathrm{I}$ or $\mathrm{U}=2.5 \mathrm{~V}-0.185 \mathrm{I}$. Therefore, the value of the current can be calculated indirectly through the motor's output voltage. As for the motor speed test circuit, the motor speed is measured by a quadrature encoder.

(3) SEMG sensor

The acquisition process of the sEMG sensor is as follows: first, the surface electrode guides the skin surface action potential; second, the preamplifier composed by three op amp filters common mode noise and amplifies the corresponding signal; third, the $50 \mathrm{~Hz}$ frequency interference is eliminated with an active dual T band-stop filter; finally, the data are put into the dSPACE after A/D sampling.

(4) DSPACE HIL simulation platform

The dSPACE HIL simulation platform is capable of real-time detection and recording of a variety of different signals, because it has wide range of information transmission interface. Its powerful data processing function provides the convenience for subsequent analysis and processing.

The control scheme is shown in Figure 1. The rehabilitation device and operator are able to exhibit human-computer interaction. During the training process, the dSPACE collects the motor's current and speed by the A/D interface. In addition, the signals of the surface EMG can be obtained from the sEMG sensor fixed to operator's upper limb by the A/D interface, achieving real-time monitoring of the training process. After the integration of information, the system can evaluate the training situation, so the program can be improved. To a certain extent, this system can offer help for the patients with movement disorders caused by stroke.

\subsection{Predictive analysis of muscle force}

In the rehabilitation system, a time domain index is used to process and analyze the sEMG signals for real-time muscle activity. Of all time domain indexes, root mean square (RMS) is most commonly used to predict muscle force using the equation given below.

$$
\mathrm{RMS}=\sqrt{\frac{1}{\mathrm{~N}} \sum_{\mathrm{i}=1}^{\mathrm{N}} \mathrm{v}_{\mathrm{i}}{ }^{2}}
$$

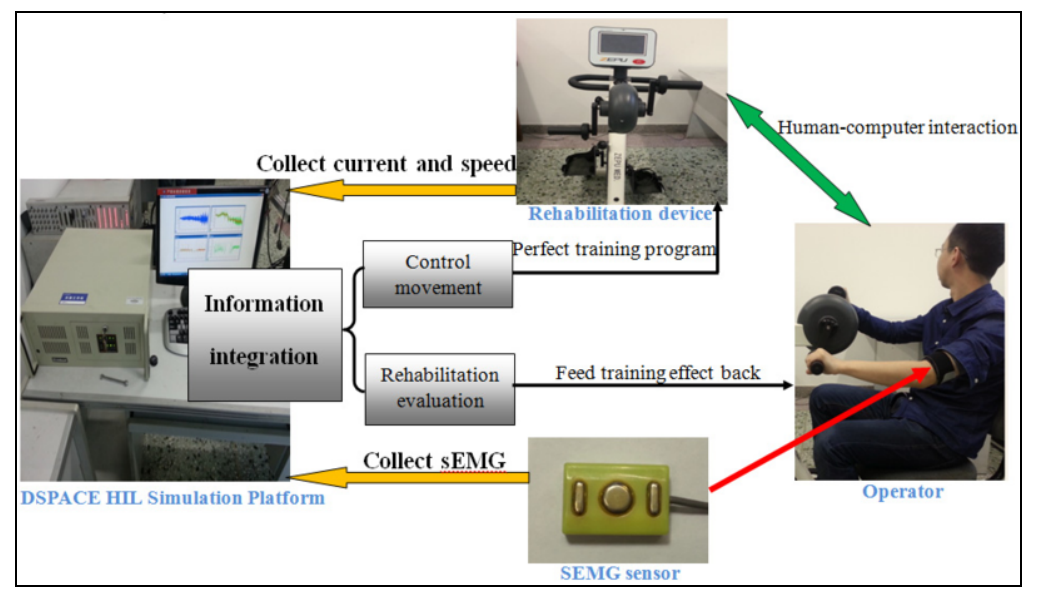

Fig. 1. The control scheme of the system. A yellow arrow means signal flow. A green arrow indicates the interaction. A red arrow points to the place of sEMG sensor. A black arrow means information feedback. 
where $\mathrm{N}$ and $\mathrm{v}_{\mathrm{i}}$ are the number of sampling points and the voltage value of the $\mathrm{i}$-th sampling point, respectively.

The sEMG measured by the sEMG sensor varies due to individual differences. So, it is necessary to establish a normalization process for sEMG. The solution is to finish maximum voluntary contraction experiments before the rehabilitation experiments to obtain the maximum sEMG. Then, the maximum RMS is calculated, which is used for the normalization process [15]. The equation is given as follows.

$$
\mathrm{RMS}_{\mathrm{N}}=\frac{\mathrm{RMS}}{\mathrm{RMS}_{\mathrm{MVC}}}
$$

where $\mathrm{RMS}_{\mathrm{N}}$, RMS and $\mathrm{RMS}_{\mathrm{MVC}}$ are the normalized RMS, the non-normalized RMS, and the benchmark RMS of sEMG, respectively.

\section{Experimental study of upper limb training}

Considering the reliability and the feasible operability of the experiment, five muscular, healthy males with normal posture were chosen as subjects [16]. Their ages were 23, 23, 25, 25, and 26. Thus, an experimental platform was created, consisting of the subject, the rehabilitation training device, and the dSPACE HIL simulation platform [17]. On the basis of the system, maximum voluntary contraction (MVC) experiments, passive training experiments under different speeds, and active training experiments under different damping are completed by each subject.

\subsection{MVC experiment}

The maximum voluntary contraction experiment must be finished before the rehabilitation experiments that obtain the maximum sEMG, which is then used for the normalization process. In the experiment, the surface electrode of the sEMG sensor was attached on the biceps' skin surface with a bandage. To obtain the maximum sEMG, a manual testing approach was used. The tester attempted to perform an elbow flexion motion against the resistance of another person. The test was repeated three times in the experiment. The results show that the maximum voluntary contraction $\mathrm{RMS}_{\mathrm{MVC}}=1.56 \mathrm{~V}$.

\subsection{Passive training experiment}

Throughout passive training, the rehabilitation device assists subjects to passively complete the corresponding action [18]. The speed of the machine's end, the motor's output torque, the motor current from five subjects, the sEMG of biceps, the degree of muscle activation, the RMS from five subjects are shown in Figure 2 when the speed is set to $20 \mathrm{r} / \mathrm{min}, 35 \mathrm{r} / \mathrm{min}$, and $50 \mathrm{r} / \mathrm{min}$.

(1) Figure 2A shows that the motion speed of upper limb increases with increasing set speed at the end of machine. This contributes to training joint activity in varying degrees by adjusting the machine's speed. In Figure 2B, under three set speed, the motor's output torque varies periodically with changes in actual speed during passive training. Moreover, the peak output torque increases slightly with the set speed increasing from $20 \mathrm{r} / \mathrm{min}$ to $35 \mathrm{r} / \mathrm{min}$ to $50 \mathrm{r} / \mathrm{min}$. That is to say, the torque of the machine's end exerting hand increases slightly. It can be concluded that passive training has less impact on strength training, despite the increasing set speed at the end of machine. 

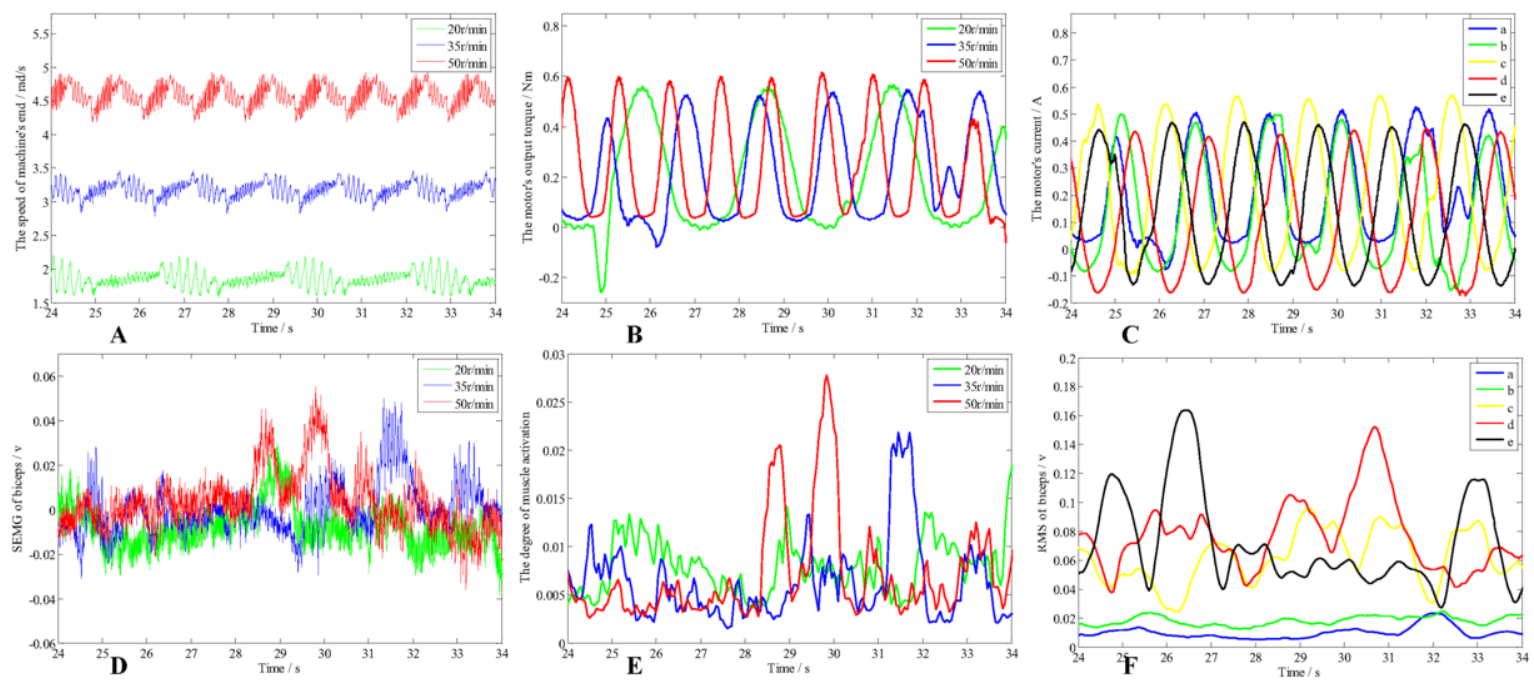

Fig. 2. The data from the process of passive training. A. The speed of machine's end. B. The motor's output torque. C. The motor current from five subjects. D. SEMG of biceps. E. The degree of muscle activation. F. RMS from five subjects.

(2) When examining both Figures 2B and 2E, it is obvious that the degree of muscle activation varies periodically with torque, revealing a direct correlation between the two in spite of its different amplitudes. Throughout the three cases, the degree of muscle activation increases slightly with increasing set speed at the end of the machine, which means that the muscle force increases slightly in the training process. However, the degree of muscle activation is smaller all the time, oscillating between 0.003 and 0.0275 . This also means that passive training has less impact on strength training, despite the increasing set speed at the end of machine.

\subsection{Active training experiment}

During active training, the subjects actively push the rehabilitation device to complete the corresponding action. The speed of the machine's end, the motor's output torque, the motor current from five subjects, the sEMG of biceps, the degree of muscle activation, the RMS from five subjects are shown in Figure 3 when the damping is set to $5 \mathrm{~N}, 10 \mathrm{~N}$, and $15 \mathrm{~N}$.

(1) In Figure 3A, the angular velocity changes slightly despite the different damping settings. This is because the damping is not large enough for the operator and it is not enough to cause a change in speed. The motor's output torque under three kinds of circumstances is shown in Figure 3B. It is easy to see that the output torque of the motor increases with increasing set damping, making the torque of machine acting on hand increase. This shows that adjusting the damping is helpful for strength training.

(2) When examining Figure 3E, it is obvious that the degree of muscle activation increases with increasing set damping, which means that the muscle force increases. Moreover, in the active training experiment, the maximal activation degree of the muscles is about 0.12 , reflecting a higher degree of muscle activation than the passive training experiment. This means that active training has better impact on strength training. 

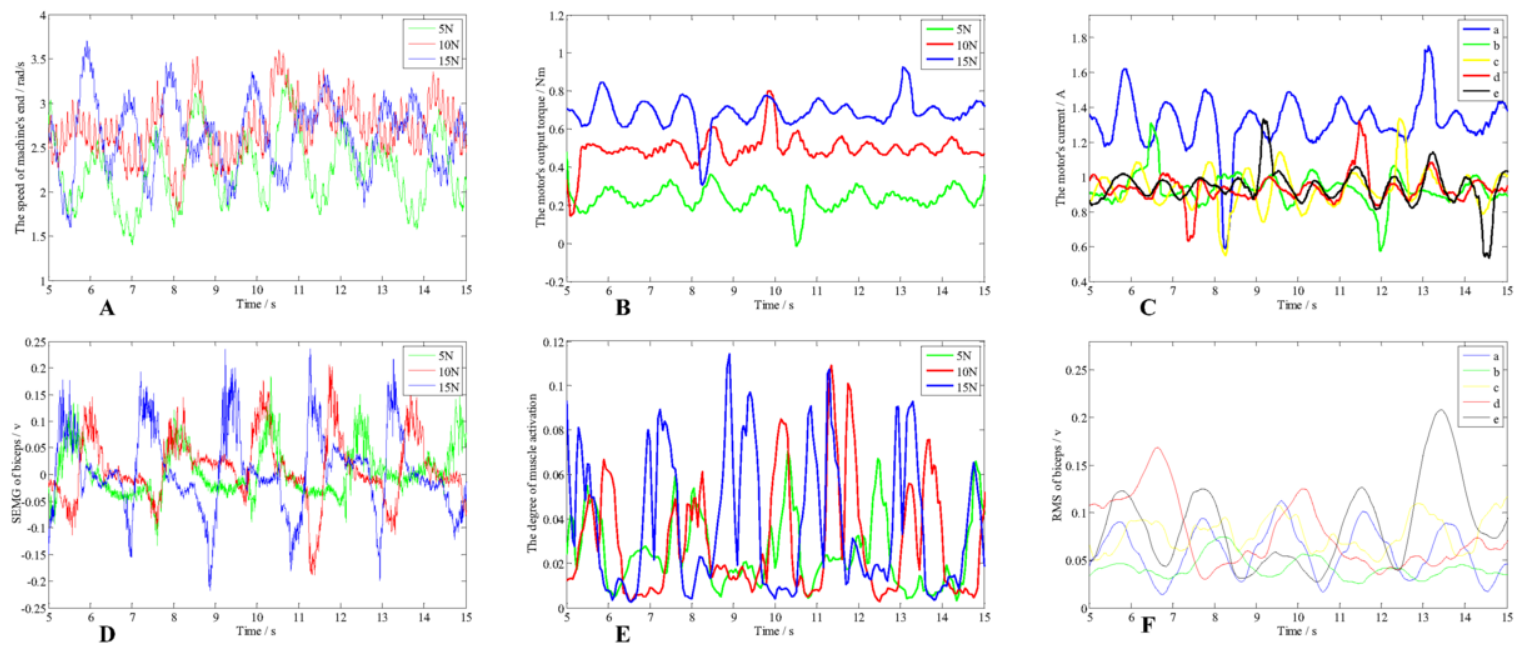

Fig. 3. The data from the process of active training. A. The speed of machine's end. B. The motor's output torque. C. The motor current from five subjects. D. SEMG of biceps. E. The degree of muscle activation. F. RMS from five subjects.

\section{Conclusions and future works}

On the basis of this system, MVC experiments, passive training experiments, active training experiments have been completed. The conclusions are given as follows.

(1) In the process of passive training, with the increasing set speed of the machine's end, the motion speed of the upper limb also increases, contributing to training joint activity by adjusting the machine speed. In addition, the degree of muscle activation increases slightly with increasing set speed, but its value is smaller all the time, oscillating between 0.003 and 0.0275 , which means that the muscle force is always smaller. This shows that passive training has less of an impact on muscle activation training.

(2) In the process of active training, the degree of muscle activation varies periodically with torque, revealing a positive correlation between the two. Moreover, the degree of muscle activation increases with increasing set damping, so the muscle force increases. The maximal activation degree of the muscles is about 0.12 , reflecting a higher degree of muscle activation than the passive training experiment. Adjusting the damping in the process of active training is helpful to train muscle strength.

In addition, the system is capable of monitoring of patients' motion information and skin surface EMG in real time. This can reflect patients' joint activity and increasing muscle strength. Patients can recover upper limb movement function quickly and more safely with the help of training programs' change in real time.

However, in the system, the value of muscle force is measured by the RMS of the sEMG when patients participate in rehabilitation training, so future work will focus on the quantitative analysis of muscle force.

\section{Acknowledgments}

This research is supported by grants from the National Natural Science Foundation of China (grants 51405095) and Harbin Foundation (grants 2014RFXXJ101). 


\section{References}

[1] A. Handley, P. Medcalf, K. Hellier and D. Dutta, Movement disorders after stroke, Age Ageing 38 (2009), 260-266.

[2] M.H. Hu, S.S. Hsu, P.K. Yip, J.S. Jeng and Y.H. Wang, Early and intensive rehabilitation predicts good functional outcomes in patients admitted to the stroke intensive care unit, Disability and Rehabilitation 32 (2010), 1251-1259.

[3] Y.F. Yi, Research on the structure design and control system of arm rehabilitative robot, Ph.D. Dissertation, Harbin Engineering University, 2011.

[4] G.C. Xu and L.L. Li, Movement function evaluation in patients with stroke, Chinese Journal of Clinical Rehabilitation 6 (2002), 1233-1235

[5] N. Hogan, H.I. Krebs, J. Charnnarong, P. Srikrishna and A. Sharon, MIT-MANUS: A workstation for manual therapy and training II, Telemanipulator Technology 1833 (1992), 28-34.

[6] H.I. Krebs, B.T. Volpe, M.L. Aisen and N. Hogan, Increasing productivity and quality of care: Robot-aided neurorehabilitation, Journal of Rehabilitation Research and Development 37 (2000), 639-652.

[7] D.J. Reinkensmeyer, L.E. Kahn, M. Averbuch, A. McKenna-Cole, B.D. Schmit and W.Z. Rymer, Understanding and treating arm movement impairment after chronic brain injury: Progress with the ARM guide, Journal of Rehabilitation Research and Development 37 (2000), 653-662.

[8] N. Friedman, J.B. Rowe, D.J. Reinkensmeyer and M. Bachman, The manumeter: A wearable device for monitoring daily use of the wrist and fingers, IEEE Journal of Biomedical and Health Informatics 18 (2004), 2168-2194.

[9] J.E. Duarte, B. Gebrekristos, S. Perez, J.B. Rowe, K. Sharp and D.J. Reinkensmeyer, Effort, performance, and motivation: Insights from robot-assisted training of human golf putting and rat grip strength, 2013 IEEE 13th International Conference on Rehabilitation Robotics, Seattle, WA, United States, 2013, pp. 1-6.

[10] R. Loureiro, F. Amirabdollahian, M. Topping, B. Driessen and W. Harwin, Upper limb robot mediated stroke therapyGENTLE/s approach, Autonomous Robots 15 (2003), 35-51.

[11] A.O. Andrade, A.A. Pereira, S. Walter, R. Almeida, R. Loureiro, D. Compagna and P.J. Kyberd, Bridging the gap between robotic technology and health care, Biomedical Signal Processing and Control 10 (2014), 65-78.

[12] Y.B. Zhang, Z.X. Wang, L.H. Ji and S. Bi, The clinical application of the upper extremity compound movements rehabilitation training robot, Proceedings of the 2005 9th International Conference on Rehabilitation Robotics, Chicago, IL, United States, 2005, pp. 91-94.

[13] X.F. Zhang, L.H. Ji and L.Y. Guo, A novel robot neurorehabilitation for upper limb motion, Proceedings of the 2005 27th Annual International Conference of the Engineering in Medicine and Biology Society, Shanghai, China, 2005, pp. 5040-5043.

[14] Q.L. Li, M.X. Kong, Z.J. Du and L.N. Sun, A novel 5-DOF exoskeletal rehabilitation robot system for upper limbs, High Technology Letters 15 (2009), 245-249.

[15]C. Chen, Analyses of EMG signal processing method and EMG assessment of occupational low back pain, Ph.D. Dissertation, Tianjin University, 2003.

[16] J.W. Qin, Z. Chen, C.Q. Li and J.J. Jia, 3 DOF upper limb rehabilitation robot-assisted training system, 2014 International Conference on Mechatronics, Electronic, Industrial and Control Engineering, Shenyang, China, 2014, pp. 365-369.

[17]L. Zhao, Comprehensive review on main technology of exoskeletal robot system for upper limb rehabilitation, Engineering Research and Designing for Industry 623 (2014), 219-225.

[18] G.Z. Xu, A. Song, L.Z. Pan, X. Gao, Z.W. Liang, J.F. Li and B.G. Xu, Clinical experimental research on adaptive robotaided therapy control methods for upper-limb rehabilitation, Robotica 32 (2014), 1081-1100. 